\title{
Sargassumilici folium (Turner) C.Agardh Ameliorate Oxidative Stress Induced Neuronal Degeneration in the Animal Model of Alzheimer's Disease
}

\author{
M. Sumithra' ${ }^{1}$ G. Arunachalam ${ }^{2}$ and V. Chitra ${ }^{1}$ \\ ${ }^{1}$ SRM College of Pharmacy, SRM University, Kattankulathur, India. \\ ${ }^{2}$ PGP College of Pharmaceutical Sciences and Research Institute, Namakkal, India. \\ http://dx.doi.org/10.13005/bbra/2134
}

(Received: 11 March 2016; accepted: 20 April 2016)

\begin{abstract}
Aluminium is a neurotoxic metal, exposure leads to neurodegenerative disease like alzheimer's. Chronic exposure to it is a real eventuality due to its presence in food, air, water, in medicines and as floculating agents in water purification process. It is an important task to control its toxicity by natural source, so the present work aimed to investigate the neuroprotective activity of ofSargassumilicifolium against aluminium induction. Aluminium chloride of dose $(100 \mathrm{mg} / \mathrm{kg}$ b.wt) was given orally to induce cognitive dysfunction in rats. The ethanolic extract and ethyl acetate fraction of dose 100, $200,400 \mathrm{mg} / \mathrm{kg}$ and $200 \mathrm{mg} / \mathrm{kg}$ respectively were selected and administered from $21^{\text {st }}$ day of aluminium induction up to 40 days and in drug treated animals behavioural studies, antioxidant studies, neurotransmitter estimation and finally histopathological studies were performed. The study has revealed the potential neuro protective effect by improving the learning memory status in behavioural studies, reversing the stress induced free radicals and boosting the neurotransmitters level responsible for memory consolidation.
\end{abstract}

Keywords: Alzheimer's disease, Neurotoxic metal, Histopathological studies.

Aluminium is a metal constitutes about $8 \%$ of the surface of the earth. Aluminium exposure disturbs central nervous system, haemopoietic and skeletal system. The neurohypothesis states that slow accumulation of aluminium in brain leads to amnesia and dementia. The theories includes imbalance between excitatory and inhibitory neurotransmitters, alternation in synaptic membrane, changes in calcium release and uptake, free radical generation and finally cytoskeleton dysfunction. ${ }^{1,2}$

Neurodegenerative diseases are estimated to surpass cancer as the second most

\footnotetext{
* To whom all correspondence should be addressed. E-mail: sumi26379@gmail.com
}

common cause of death among elderly by the $2040^{3}$. For this reason, a great deal of attention has been expressed by scientists regarding safe and effective neuroprotective agents. ${ }^{4}$

Marine algae represent one potential candidate neuroprotective agent. However, development of marine algae as neuroprotective agents still faces several challenges. The rationale for marine algal neuroprotective effects treatment in the CNS is based on established observations and experiments in vitro or in animal models only. Up to now, none of the marine algal neuroprotective effects have been examined in human subjects. Therefore, small clinical studies and further largescale controlled studies are needed. diseases. Marine algae are an important source of bioactive ingredients that can be applied to many aspects of 
processing healthier foods and developing functional neuroprotective foods. ${ }^{5}$

\section{MATERIALAND METHODS}

\section{Experimental design of aluminium induced cognitive dysfunction}

Animals were divided in to seven groups of six rats in each group and all experiments were conducted at the same time (9.00A.M) of the day to minimize circardian influence. The experimental protocols were approved by the institutional ethics committee XIII/ VELS/PCOL/51/2000/CPCSEA/ IAEC/8.8.12. All groups except control were treated with aluminium chloride $(100 \mathrm{mg} / \mathrm{kg})$ once daily for a period of 40days.From the $21^{\text {st }}$ day , extract, fraction were administered to animals post orally upto 40 days.

Group-1 served as control; Group-2 treated as aluminium chloride (100mg/kg b.wt)

Group-3 was treated with aluminium chloride $(100 \mathrm{mg} / \mathrm{kg}$ b.wt $+100 \mathrm{mg} / \mathrm{kg}$ b.wt of EESI

Group-4 treated with aluminium chloride (100mg/ $\mathrm{kg}$ b.wt) + 200mg/kg b.wt of EESI

Group-5 treated with aluminium chloride (100mg/ $\mathrm{kg}$ b.wt)+400mg/kg b.wt of EESI

Group-6 aluminium chloride (100mg/kg b.wt)+ Ethyl acetate fraction of (200mg/kg b.wt)

Group-7 treated with aluminium chloride (100mg/ kg b.wt) + piracetam (200mg/kg b.wti.p)

\section{Chemicals and Instruments}

Rectangular arm maze and Poles climbing apparatus-Orchid Scientific supplier, Nashik Pune.

Aluminium chloride from Southern scientific suppliers, Piracetam tablet from medical shop, antioxidant chemicals from Sigma Aldrich, dopamine and serotonin as gift sample from Biocon pharmaceuticals, Banglore.

\section{Behavioural studies \\ Rectangular arm maze}

The arm maze was constructed with two chambers (A and C) and a central corridor(B) partitioned by wooden slits. The learning process was conducted by placing food item in reward chamber. Rats were trained to reach the reward chamber by passing the corrider was considered as the final stage of the training. Training session was conducted twice a day for four days (From $35^{\text {th }}-39^{\text {th }}$ day) and on the last day retention memory was noted. ${ }^{6}$

\section{Passive avoidance paradigm}

This method was based on the negative reinforcement to study the long term memory. Cognitive impairment was studied by poles climb apparatus with the index of step down latency (SDL).$^{7-9}$

\section{Antioxidant studies}

The rats used for rectangular arm maze were used for antioxidant studies. Rats were sacrificed by cervical dislocation, brain was isolated, homogenised with an addition of 10 volumes of sterile normal saline and centrifuged at 3000rpm for $10 \mathrm{~min}$ and the resultant used for the following study

\section{Lipid Peroxidation}

The lipid peroxidation was determined by Devasagayam et al 1987. The amount of MDA formed / $\mathrm{mg}$ protein is used to express the level of lipid peroxidation. ${ }^{10}$

\section{Superoxide Dismutase (SOD)}

In whole brain the SOD was determined by Marklund S et al.,1997.Take $0.5 \mathrm{ml}$ of brain homogenate, to it $0.25 \mathrm{ml}$ of absolute ethanol , 0.15 $\mathrm{ml}$ of chloroform were added, shaked for 15 minutes and centrifuged. To $2 \mathrm{ml}$ of the supernant, $2.5 \mathrm{ml}$ of $0.1 \mathrm{M}$ TrisHCl and $1.5 \mathrm{ml}$ of pyrogallol were mixed and the resultant was measured at $470 \mathrm{~nm}$ for every 1 min upto 3 mins. ${ }^{11}$

\section{Catalase}

In the brain the catalase enzyme activity was estimated by Sinha et al., 1972.The colour changes was measured at $570 \mathrm{~nm}$ using colorimeter. ${ }^{12}$

\section{Glutahione peroxidase (GPx)}

GPx estimation was performed as mentioned by Rotruck et al., 1973. The colour developed was read at $420 \mathrm{~nm}$. Glutathione peroxide activity was expressed as mg of glutathione utilized / min /mg protein under incubation conditions. ${ }^{13}$

Glutathione - S - Transferse (GST)

GST in whole brain was estimated by .Habiget al 1974.The change in optical density and measured at $340 \mathrm{~nm}$ using a colorimeter.The enzyme activity was expressed as $\mathrm{m}$ moles of CDNB conjugated / min /mg protein under incubation conditions. ${ }^{15}$

\section{Histopathological studies}

Rats used for passive avoidance paradigm were sacrificed and in each group a brain sample was subjected to histopathological studies. 


\section{Estimation of neurotransmitter}

Rats used for passive avoidance paradigm, were sacrificed and the brain were isolated to prepare homogenate as mention in antioxidant studies.

\section{Preparation of tissue extract}

$1 \mathrm{gm}$ of brain homogenate with $3 \mathrm{ml}$ HClbutanol, centrifuged for 10 minutes at $2000 \mathrm{rpm}$. To $0.8 \mathrm{ml}$ of supernatant , $2 \mathrm{ml}$ of heptane, $0.25 \mathrm{ml}$ $0.1 \mathrm{M}$ of $\mathrm{HCl}$, after 10 minutes, the tube was centrifuged under the same condition to separate the two phases. Upper organic phase for serotonin estimation and the aqueous phase for dopamine assay. ${ }^{16}$

\section{Estimation of Dopamine level}

To $0.02 \mathrm{ml} \mathrm{HCl}$ phase $+0.005 \mathrm{ml} 0.4 \mathrm{M} \mathrm{HCl}$ , $0.01 \mathrm{ml}$ EDTA buffer, $1 \mathrm{ml}$ iodine solution., $0.1 \mathrm{ml}$ sodium thiosulphate in $5 \mathrm{M}$ sodium hydroxide, 0.1 $\mathrm{ml}$ of $10 \mathrm{M}$ acetic acid were added then heated to $100^{\circ} \mathrm{C}$ for $6 \mathrm{~min}$. The resultant measured at 330 to $375 \mathrm{~nm}$ using spectrofluorimeter.

\section{Estimation of Serotonin Assay}

$1 \mathrm{ml}$ of organic supernatant phase, and $0.2 \mathrm{ml}$ of heptane, $0.025 \mathrm{ml}$ of $0.1 \mathrm{M} \mathrm{HCl}$ were centrifuged for $10 \mathrm{mins}$, then separate aqueous phase of $0.25 \mathrm{ml}, 0.025 \mathrm{ml}$ of O-phthalaldehyde was mixed and heated at $100^{\circ} \mathrm{C}$ for 10 mins. The Flourescence emitted was measured at 360-470nm.

$$
\mathrm{C}_{\mathrm{u}=} \mathrm{C}_{\mathrm{s}} \mathrm{XF}_{\mathrm{u}}
$$

C concentraation of standard

$\mathrm{F}_{u}^{\mathrm{s}-}$ Flourescence intensity of unknown

$\mathrm{F}_{\mathrm{s}}$ - Flourescenceintensity of unknown

\section{RESULTAND DISCUSSION}

\section{Rectangular arm maze}

In rectangular arm maze, the transfer latency was determined and the results were mentioned in table-1.The negative control group showed significant increase in transfer latency period compared to normal control implies the loss of memory but in extract and fraction treated group there is a decrease in transfer latency period showed the memory enhancing capacity of the extract and in high dose of extract and ethyl acetate fraction treated groups results were equivalent to standard drug treated group.

Passive avoidance paradigm

In this task, punishment given to animals inorder to learn the task forcefully and the step down latency period was noted, the difference in latency period were tabulated in table-2.The control animal retention memory is noticed by increase in time period to step down wheras in aluminium chloride treated group due to memory deficit animal deliberately steps down but in case of extract and fraction treated group step down latency is increased proved the memory enhancing ability of Sargassumilicifolium.

\section{Antioxidant studies}

The role of oxidative stress in amnesia is well known which further increases the level of stress in dementia and AD. The antioxidant effect of extract and fraction of Sargassumilicifolium Turner (C.Agardh) was studied and mentioned in Table-3.The extract treated group reverses the lipid peroxidation compared to aluminium chloride induced group, and boost the antioxidant levels.

\section{Histopathological studies}

In normal control animals, neuronal cells were active and relatively packed with prominent nuclei. In aluminium chloride treated group, spongy cell, neuronal necrosis, cells degenerated with small nuclei leading to plaque formation. In EESI 100mg, $200 \mathrm{mg} / \mathrm{kg}$ few cells undergo neuronal decongestion whereas EESI400mg/kg, EFSI 200mg/ kg showed significant improvement and in both the groups there is no cell degeneration with prominent nuclei. In piracetam treated group there was no neuronal degeneration and cells were similar to normal control group.

\section{Estimation of Neurotransmitters}

The role of neurotransmitters like dopamine and serotonin in learning and memory process were monitored and results mentioned in Table-4.In aluminium treated group, there is a significant decrease in serotonin and dopamine level controlled to normal group wheras in low dose treated group there is no improvement but in medial dose and fraction treated group there is a slight progress and in high dose treated group prominent increase in neurotransmitter levels which was equivalent to standard group implies the phytoconstituent present in the extract were merely responsible for memory consolidation process by preventing neurotransmitter degeneration. Neurotransmitter alternations are commonly regarded as epiphenomena, secondary to neuronal death and the tissue reaction to it . The levels of 
Table 1. Effect of Sargassumilicifolium (Turner) C.Agardh on transfer latency (Rectangular arm maze) in aluminium chloride induced amnesia

\begin{tabular}{llll}
\hline Group & Treatment & $\begin{array}{l}\text { Transfer latency } \\
\text { (sec)Acquisition period }\end{array}$ & $\begin{array}{l}\text { Transfer latency } \\
\text { (sec)Retention mremory }\end{array}$ \\
\hline I & Normal & $90.50+8.06$ & $89.66+20.94$ \\
II & Scopolamine & $168.28+26.50^{\#}$ & $185.16+17.07^{\# \#}$ \\
III & EESI 100mg/kg & $85.02+12.80^{*}$ & $91.84+28.32^{*}$ \\
IV & EESI 200mg/kg & $62.54+5.48^{\text {ns }}$ & $59.88+4.32^{*}$ \\
V & EESI 400mg/kg & $45.50+3.28^{* *}$ & $42.83+4.80^{*}$ \\
VI & EFSI200mg/kg & $69.20+7.89^{*}$ & $69.84+29.32^{*}$ \\
VII & Piracetam-200mg & $20.52+3.80^{* *}$ & $16.62+2.88^{*}$ \\
\hline
\end{tabular}

All the datas were analysed by one way ANOVA followed by Dunnetts $\mathrm{T}$ test.Values were expressed by mean +sem. Group-3,4,5,6,7 were compared with group -2 whereas Group2 was compared with group -1 and represented by $\mathrm{P}^{*}<0.05$ : $\mathrm{P}^{* *}<0.01 . \mathrm{P}^{\#}<0.05$ respectively

Table 2. Effect of Sargassumilicifolium (Turner) C.Agardh on step down latency (Passive avoidance paradigm) in aluminium chloride induced amnesia

\begin{tabular}{llll}
\hline Group & \multirow{2}{*}{ Treatment Groups } & \multicolumn{2}{c}{ Step down latency(sec) } \\
& & Acquisition period & Retentive memory \\
\hline I & Normal & $6.15+0.70$ & $7.42+1.08$ \\
II & Scopolamine & $1.83+0.30^{\#}$ & $2.66+2.23^{\#}$ \\
III & EESI 100mg/kg & $8.01+0.74^{*}$ & $12.5+1.22^{*}$ \\
IV & EESI 200mg/kg & $11.83+1.38^{*}$ & $14.66+3.41^{*}$ \\
V & EESI 400mg/kg & $16.5+1.25^{*}$ & $21.96+1.58^{* *}$ \\
VI & EFSI200mg/kg & $9.22+1.24^{*}$ & $11.96+1.24^{*}$ \\
VII & Piracetam 200mg/kg & $23.02+1.46^{*}$ & $31.34+2.62^{* *}$ \\
\hline
\end{tabular}

All the datas were analysed by one way ANOVA followed by Dunnetts $T$ test.Values were expressed by mean +sem. Group-3,4,5,6,7 were compared with group -2 whereas Group-2 was compared with group- 1 and represented by $\mathrm{P}^{*}<0.05$ : $\mathrm{P}^{* *}<0.01$. $\mathrm{P}^{\#}<0.05$ respectively.

Table 3. Effect ofSargassumilicifolium (Turner) C.Agardh on antioxidant studies

\begin{tabular}{|c|c|c|c|c|c|c|}
\hline \multicolumn{2}{|c|}{ S.No Treatment } & \multirow{2}{*}{$\begin{array}{c}\mathrm{LPO}^{\mathrm{a}} \\
16.98+0.86\end{array}$} & \multirow{2}{*}{$\frac{\mathrm{SOD}^{\mathrm{b}}}{66.30+1.28}$} & \multirow{2}{*}{$\begin{array}{l}\text { Catalase }^{c} \\
72+2.58\end{array}$} & \multirow{2}{*}{$\frac{\mathrm{GPX}^{\mathrm{d}}}{32.66+1.24}$} & \multirow{2}{*}{$\frac{\mathrm{GST}^{\mathrm{e}}}{28.66+2.52}$} \\
\hline 1 & Normal & & & & & \\
\hline 2 & $\mathrm{Alcl}_{3}$ induced & $28.64+1.78^{\# \#}$ & $45.04+2.26^{\#}$ & $49.02+3.12^{\mathrm{ns}}$ & $25.64+1.86^{\#}$ & $16.44+0.95^{\#}$ \\
\hline 3 & $\mathrm{Alcl}_{3}^{3}+$ EESI 100mg/kg b.wt & $25.08+6.86 *$ & $46.54+2.27^{*}$ & $52.62+2.56^{*}$ & $26.22+2.04^{* *}$ & $17.98+1.32^{*}$ \\
\hline 4 & $\mathrm{Alcl}_{3}+$ EESI 200mg/kg b.wt & $24.98+2.82^{\mathrm{ns}}$ & $47.02+3.30^{*}$ & $55.08+1.88^{*}$ & $27.08+2.11^{*}$ & $19.64+4.02^{* *}$ \\
\hline 5 & $\mathrm{Alcl}_{3}^{3}+$ EESI 400mg/kg b.wt & $19.28+4.64^{* *}$ & $49.28+2.70^{* *}$ & $58+2.34^{* *}$ & $30.04+2.64^{*}$ & $21.66+1.89^{*}$ \\
\hline 6 & $\mathrm{Alcl}_{3}+\mathrm{EFSI} 200 \mathrm{mg} / \mathrm{kg}$ b.wt & $18.96+6.08^{*}$ & $52.34+1.72^{*}$ & $61.62+1.64^{*}$ & $29.62+1.88^{*}$ & $23.02+3.04^{*}$ \\
\hline 7 & $\mathrm{Alcl}_{3}^{3}+$ Piracetam & $15.54+3.98^{*}$ & $63.28+1.52^{* *}$ & $68.22+1.98^{* *}$ & $31.54+2.44^{*}$ & $24.22+1.58^{*}$ \\
\hline
\end{tabular}

All the datas were analysed by one way ANOVA followed by Dunnetts $\mathrm{T}$ test.Values were expressed by mean + sem. Group-3,4,5,6,7 were compared with group -2 whereas Group-2 was compared with group-1 and represented by $\mathrm{P}^{*}<0.05: \mathrm{P}^{* *}<0.01 . \mathrm{P}^{\#}<0.05$ respectively 
Table 4. Estimation of neurotransmitters on Sargassumilicifolium (Turner) C.Agardh

\begin{tabular}{llll}
\hline S.No & Treatment schedule & Serotonin (ng/mg of tissue) & Dopamine(ng/mg of tissue) \\
\hline 1 & Normal & $6.7+0.76$ & $18.75+1.76$ \\
2 & Alcl $_{3}$ induced & $3.28+0.24^{\#}$ & $10+2.02^{\#}$ \\
3 & Alcl $_{3}+$ EESI 100mg/kg b.wt & $3.70+0.22^{*}$ & $9.25+8.04^{\text {ns }}$ \\
4 & Alcl $_{3}+$ EESI 200mg/kg b.wt & $4.06+01.08^{* *}$ & $10.75+0.03^{\text {ns }}$ \\
5 & Alcl $_{3}+$ EESI 400mg/kg b.wt & $5.02+1.22^{*}$ & $14.2+6.06^{* *}$ \\
6 & Alcl $_{3}$ induced+EFSI200mg/kg & $4.37+2.98^{*}$ & $12.25+8.64^{*}$ \\
7 & Alcl3+ Piracetam & $5.50+3.10^{*}$ & $16.4+8.44^{* *}$ \\
\hline
\end{tabular}

All the datas were analysed by one way ANOVA followed by Dunnetts $\mathrm{T}$ test.Values were expressed by mean +sem. Group-3,4,5,6,7 were compared with group -2 whereas Group-2 was compared with group-1 and represented by $\mathrm{P}^{*}<0.05$ : $\mathrm{P}^{* *}<0.01 . \mathrm{P}^{\#}<0.05$ respectively.ns - represent the probility was not signifant when compare to aluminium control.

Histopathology of cerebral cortex in aluminium induced amnesia Normal Alcl3 treated group EESI 100mg/kg EESI 200mg $/ \mathrm{kg}$ EESI $400 \mathrm{mg} / \mathrm{kg}$ EFSI200mg/kg Piracetam
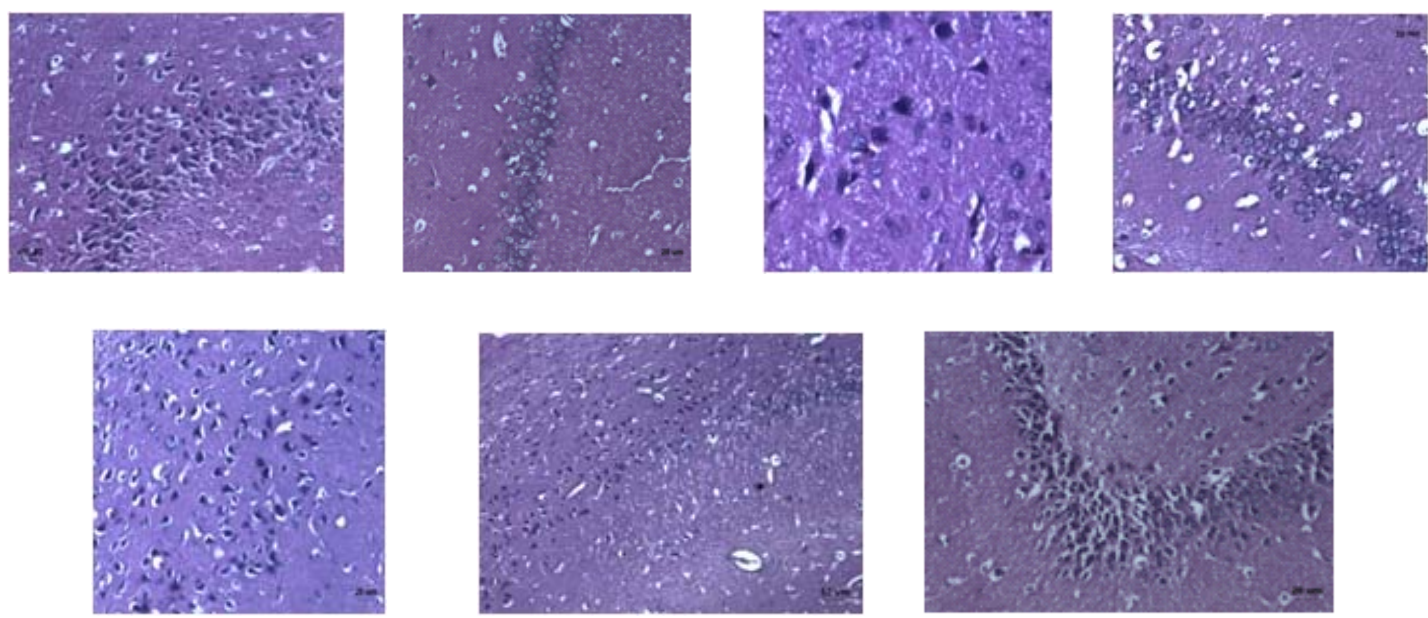

neurotransmitters and neuropeptides are markedly affected during amnesia and AD. Among the neurotransmitters, dopamine, serotonin and acetyl choline has an important role in learning and memory. Brain neurochemical changes purely associated with alzheimers disease and amnesia. Currently approved therapies for AD depends primarily on manipulating the cholinergic system, to act at the brain by increasing functional levels of the excitatory transmitter. ${ }^{17}$
In linking the involvement of extracts on behavioral, antioxidant and histopathology study conflicts the cognitive function of Sargassumilicifolium against $\mathrm{AlCl}_{3}$ induction model.

\section{CONCLUSION}

To our knowledge this is the first report providing evidence for the memory enhancing 
effect of Sagassumilicifolium TurnerC.Agardh. Our data provide substantial initial evidence for the therapeutic potential and inviting further investigations. It will be interesting in future to dissect out the kinetics of individual components in the fraction and to strengthen the phytomolecule role by insilico studies.

\section{ACKNOWLEDGEMENT}

Our sincere thanks to Dean Dr.K.S.LakshmiaandDr.Parivendhen, Chancellar of SRM University, Kattangulathur for his inspiration and moral support for the success of the project.

\section{REFERENCES}

1. Alfrey A.C., Legendre G.R., Kahny W.D. The dialysis encephalopathy syndrome, Possiblealuminium intoxication. N. Engl.J. Med. ,1976; 294, 184-88.

2. Boegman R.J. and Bates L.A. Neurotoxicity of aluminium. Can.J. Physiol. Pharmacol.1984; 62, 1010-1014.

3. Bjarkam, C.R., Sørensen, J.C., Sunde, N.Å.; Geneser, F., Østergaard, K. New strategies for the treatment of Parkinson's disease hold considerable promise for the future management of neurodegenerative disorders. Biogerontology 2001, 2, 193-207.

4. Ansari, J., Siraj, A. Inamdar, N. Pharmacotherapeutic approaches of Parkinson's disease. Int. J. Pharmacol. 2010, 6, 584-590.

5. Narang S. Gibson, D., Wasan A.D., Ross, E.L., Michna, E., Nedeljkovic S.S. Jamison R.N. Efficacy of dronabinol as an adjuvant treatment for chronic pain patients on opioid therapy. $J$. Pain 2008, 9, 254-264.

6. Nayak P. Aluminium: Impacts and disease. Environ .Res, Sec.-A, 2009; 89, 111-115.

7. Indumathy S, Kavimani S, RamanK.V. Role of angiotensin antagonists in memory enhancement. International Journal of Pharma and biosciences. 2010: 1(3): 121-126.

8. Parle $\mathrm{M}$, Dhingra D and Kulkarni S K. Improvement of mouse memory by Myristicafragrans seeds. J Med Food, 2004, 7: 157-161.

9. Parle $\mathrm{M}$ and Dhingra D. Ascorbic acid: A promising memory enhancer in mice. Journal of Pharmacological sciences. 2003, 93: 129-135.

10. Devasagayam TPA, Tarachand U. Decreased lipid peroxidation in the rat kidney during gestation. BiochemBiophys Res Commun., 1987, 56: 836 -842.

11. Marklund S, Marklund G. Involvement of superoxide anion radical in the auto oxidation of pyrogallol and a convenient assay for superoxide dismutase. European JBiochem, 1997, 47: 469474.

12. Sinha AK. Colorimetric assay of catalase.Analytical Biochemistry, 1972, 47: 389394.

13. Rotruck JT, Pope AL, Ganther HL, Swanson AB. Selenium: Biochemical role as a component of glutathione peroxidase. Sci., 1973: 179: 58859.

14. Habig WH, Pabst MJ, Jakoby WB. Glutathion $\mathrm{S}$ - transferase, the first enzymatic step in mercapturic acid formation. J BiolChem, 1974: 249, 7130-7139.

15. Dilip K P. Determination of brain biogenic amines in CynodonDactylon Pers. and CyperusRotundus L. treated mice. Int J Pharm PharmSci 2009; 1: 190-197.

16. Zattal PF, Nicolini B. Corain .Aluminium (III) toxicity and blood brain barrier Permiability: IN Aluminium in Chemistry Biology and Medicine Cortina International. Verona and Reven Press, New York. 1991: 97-112.

17. Bolla KI, Briefel G, Spector D, Schwartz L, Wieler J, Herron L. Gimenez. Neurocognitive effects of aluminium. Arch . Neurol., 1992: 49: 1021-1026. 